\title{
BMJ Open Relationship between hormonal contraceptives and sleep among women of reproductive age: a systematic review protocol
}

\author{
Jinhui Ma (D) , ${ }^{1}$ Megan Cheng (D) , ${ }^{2}$ Lehana Thabane (D), ${ }^{1}$ Caihong Ma (D) , ${ }^{3}$ \\ Ning Zhang, ${ }^{4,5}$ Qi Wang, ${ }^{1}$ Hyunwoo Kim, ${ }^{1}$ Hameed Reza, ${ }^{6}$ Chunxue Wang, ${ }^{4,5}$ \\ Xiaomei Yao (D) ${ }^{1,7}$
}

To cite: Ma J, Cheng M, Thabane L, et al. Relationship between hormonal contraceptives and sleep among women of reproductive age: a systematic review protocol. BMJ Open 2021;11:e045819. doi:10.1136/ bmjopen-2020-045819

- Prepublication history for this paper is available online. To view these files, please visit the journal online (http://dx.doi. org/10.1136/bmjopen-2020045819).

Received 14 0ctober 2020 Accepted 24 September 2021

Check for updates

(c) Author(s) (or their employer(s)) 2021. Re-use permitted under CC BY-NC. No commercial re-use. See rights and permissions. Published by BMJ.

For numbered affiliations see end of article.

Correspondence to Dr Xiaomei Yao; yaoxia@mcmaster.ca and Dr Ning Zhang; 827582777@qq.com

\section{ABSTRACT}

Introduction The aetiology of sleep disruptions is unknown, but hormonal fluctuations during the menstrual cycle, pregnancy and menopause have been shown to potentially affect how well a woman sleeps. The aim of this systematic review was to investigate whether hormonal contraceptives are associated with a decreased quality of sleep and increased sleep duration in women of reproductive age.

Methods This review will analyse data from randomised controlled trials or non-randomised comparative studies investigating the association between hormonal contraceptives and sleep outcomes among women of reproductive age. Reviews addressing the same research question with similar eligibility criteria will be included. A literature search will be performed using the MEDLINE, Embase and Cochrane Central Register of Controlled Trials databases from inception to 7 March 2021. The Cochrane Collaboration's Risk of Bias for Randomised Trials V.2.0 and The Risk of Bias for Non-randomised Studies of Interventions tool will be used to assess risk of bias for each outcome in eligible studies. Two reviewers will independently assess eligibility of studies and risk of bias and extract the data. All extracted data will be presented in tables and narrative form. For sleep measures investigated by two or more studies with low heterogeneity, we will conduct random-effects meta-analysis to estimate the magnitude of the overall effect of hormonal contraceptives. If studies included in this systematic review form a connected network, a network meta-analysis will be conducted to estimate the comparative effect of different contraceptives. The Grading of Recommendations, Assessment, Development, and Evaluation approach will be used to summarise the quality of evidence. Our protocol follows the Preferred Reporting Items for Systematic Reviews and MetaAnalyses for Protocols 2015 guidelines.

Ethics and dissemination Ethics approval is not required as data were sourced from previously reported studies. The findings of this review will be published in a peer-reviewed journal and presented at relevant conferences.

PROSPERO registration number CRD42020199958.
Strengths and limitations of this study

- This systematic review on the association between hormonal contraceptive use and sleep is based on a robust, librarian consulted search strategy.

- The literature review, data extraction and risk of bias assessment are performed by two independent reviewers to mitigate bias.

- The risk of bias of each outcome from included papers will be assessed with the appropriate tool.

- Papers in languages other than English are not included, leading to potential for publication bias.

\section{INTRODUCTION}

Worldwide, there are around 1.9 billion women of reproductive age. Hormonal contraceptives, including oral contraceptives (OCs), vaginal ring, contraceptive skin patches, implants, injections and hormonal intrauterine contraceptive devices (IUDs), are widely used around the world in women of reproductive age to avoid unintentional pregnancy. ${ }^{1}$ Of the 842 million women using modern forms of contraception, $43 \%$ are using hormonal contraceptives. The overall number of users of hormonal contraceptives is increasing annually. ${ }^{1}$ One hundred and fifty-one million women use OCs with usage varying by region. ${ }^{1}$ Other hormonal contraceptives such as implants, vaginal rings and intrauterine devices have been widely adopted as well and used by over 256 million women in the world. ${ }^{1}$ These hormonal contraceptives have different dosage, formulation, mechanism of action and applicable groups compared with OCs. ${ }^{2}$ Unlike OCs which are taken consistently and provide a stable dosage of the oestrogen and progestin components, other hormonal contraceptives have the potential to provide lower dose of progestin and oestrogen. It has been shown 
that hormonal IUDs release levonorgestrel directly into the uterus; only a small amount is absorbed into the rest of the body; thus, its effects are mostly paracrine rather than systemic. ${ }^{3}$

Progesterone, a primary component in hormonal contraceptives, is an agonist of gamma-aminobutyric acid (GABA) receptors through a metabolite, allopregnanolone. ${ }^{4}$ GABA is a crucial molecule in sleep promotionmost sleep-promoting neurons are sensitive to GABA. ${ }^{5}$ Common sleep medications, such as benzodiazepines, have been shown to positively modulate GABA signalling. ${ }^{5}$ The potency of progestin is comparable to that of benzodiazepines and has similar agonistic effects. Changes in electroencephalogram activity seen with progesterone administration are similar to those evoked by benzodiazepines. ${ }^{4}$ Studies in rat models have shown that administration of exogenous progesterone leads to dose-dependent decreased sleep latency and wakefulness. ${ }^{4}$ The mechanism behind the effect of oestrogen on sleep is not well elucidated but rat studies have shown that administration of oestrogen promotes sleep during the sleep period and reduces sleep during the wakefulness period. ${ }^{6}$ The overall effect on sleep is likely caused by a combination of both oestrogen and progesterone, although the magnitude of their contribution to sleep changes is unknown. ${ }^{7}$

High-quality sleep is as essential as regular exercise and eating a balanced diet for maintenance of optimal health and well-being. Severe sleeping problems, such as insomnia, are important matters from both a public health perspective and an individual level. Insomnia is associated with depression, anxiety, substance abuse, cognitive impairment, metabolic disorders (eg, diabetes, dyslipidaemia and obesity) and cardiovascular diseases. ${ }^{8-10}$ Women are more likely than men to have sleep problems, including insomnia and restless leg syndrome. The exact aetiology of these sleep disruptions is unknown, but hormonal fluctuations during the menstrual cycle, pregnancy and menopause have been shown to potentially affect how well a woman sleeps. ${ }^{11-13}$ During ovulation, there is a surge of luteinising hormone (LH) and follicle-stimulating hormone (FSH) which leads to a decrease in oestrogen concentration and an increase in progesterone. ${ }^{14}$ During the luteal phase, progesterone concentrations increase and oestrogen levels are high up to the mid-phase. Starting from the mid-luteal phase, both oestrogen and progesterone levels decrease. ${ }^{14}$ Sleep disturbances are most commonly reported at the end of the luteal phase and early follicular phase. ${ }^{15}$ OCs are taken each day for 21 days, providing a constant, exogenous source of oestrogen and progesterone, preventing the release of FSH and LH and thus the production of a follicle or ovulation. ${ }^{16}$ Therefore, hormonal contraceptives may affect women's sleep-wake cycle and cause physiological changes that lead to sleep disturbance.

Unlike other commonly prescribed drugs, hormonal contraceptives are used by healthy women for long periods of time. Safety trials of hormonal contraceptives have focused largely on breast cancer and venous thromboembolism risk. ${ }^{17-19}$ Despite decreases in oestrogen concentration with later OC generations, its use has been shown to be associated with an increased risk of developing breast cancer and venous thromboembolism. ${ }^{20}$ A 2017 cohort study found OC intake was associated with a reduced risk of colorectal, endometrial and ovarian cancers and increased risk of cervical and breast cancers. ${ }^{21}$ Studies in women have varied findings on the association between hormonal contraceptive use and sleep quality and duration. Differences in methodology, including the formulation of contraceptives taken, stratification of formulations, measurements of sleep or the phase of the menstrual cycle of naturally cycling women used as comparison may explain the different findings between studies. For instance, a pilot study published in 2020 by Guida et al found significantly increased sleep duration for users of oral progestins, oral gestodene and $13.5 \mathrm{mg}$ levonorgestrel intrauterine systems, compared with naturally cycling women (women not currently using any hormonal contraceptive), and decreased sleep latency and better sleep quality for users of depot-administered contraceptives. ${ }^{22}$ However, a polysomnography study published in 2012 by Hachul et al found decreased sleep latency in women using OC and no difference in overall sleep quality. ${ }^{23}$ A randomised control trial by Lundin et al found no significant sleep related results, while a cross-sectional analysis by Bezerra et al found that hormonal contraceptive users had worse sleep quality compared with their naturally cycling counterparts. $^{2425}$

Given the high prevalence of hormonal contraceptive use among women of reproductive age and the relationship between hormonal contraceptive use and sleep varying in both magnitude and direction in the current literature, there is an urgent need to provide women with accurate, evidence-based information to inform their use of hormonal contraception. ${ }^{22-25}$ Hence, the objective of this systematic review was to compile and elucidate the association between hormonal contraceptive use and sleep (including the quality of sleep, sleep disorders and duration of sleep). Our hypothesis is hormonal contraceptives are associated with a decreased quality of sleep and increased sleep duration in women of reproductive age. Findings from this review will provide insights to women choosing between different methods and formulations and also inform the development of new progestins for future generations of hormonal contraceptives. The systematic review protocol was registered at the website of the International Prospective Register of Systematic Reviews.

\section{METHODS}

Our protocol follows the Preferred Reporting Items for Systematic reviews and Meta-Analyses for Protocols 2015 (PRISMA-P 2015) guidelines. $^{26}$ 
Table 1 Search strategy for OVID MEDLINE

\begin{tabular}{|c|c|}
\hline Number & Searches \\
\hline 1 & 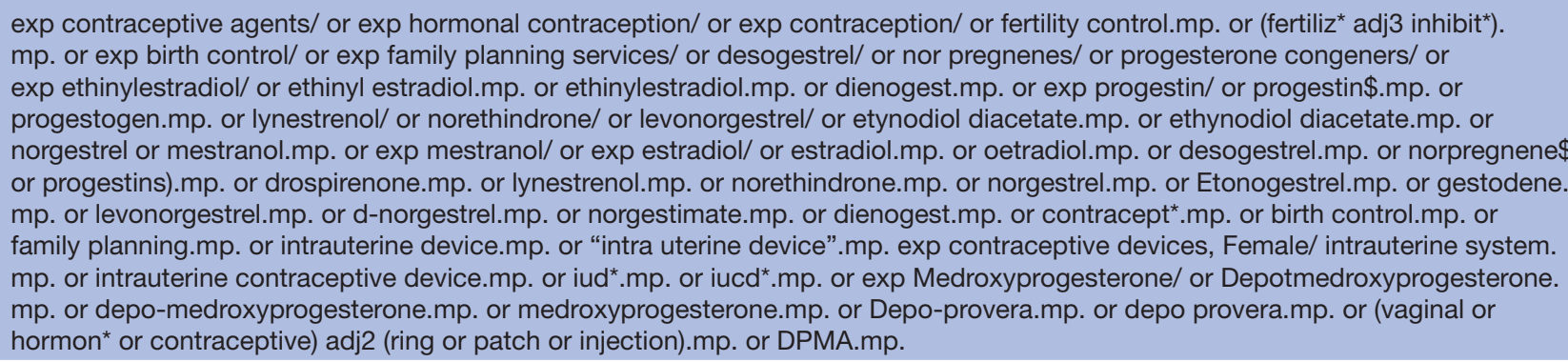 \\
\hline 2 & $\begin{array}{l}\text { Exp sleep/ or exp sleep-wake transition disorders/ or exp sleep deprivation/ or exp sleep wake disorders/ or exp dyssomnias/ or } \\
\text { fragmented sleep.mp. or insufficient sleep syndrome\$.mp. or exp sleep latency/ or exp sleep hygiene/ or exp sleep arousal disorder } \\
\text { or exp sleep disorder/ or sleep*.af. or insomnia } \$ . m p \text {. or hypersomnia\$.mp. or "disorders of excessive somnolence" or exp restless } \\
\text { legs syndrome/ or restless legs syndrome.mp }\end{array}$ \\
\hline 3 & $\begin{array}{l}\text { (comment or letter or editorial or note or erratum or short survey or news or newspaper article or patient education handout or case } \\
\text { reports or historical article).pt. }\end{array}$ \\
\hline 4 & Animal/ not human/ \\
\hline 5 & 3 or 4 \\
\hline 6 & 1 and 2 not 5 \\
\hline 7 & Limit 6 to $y r=" 1970$-current" \\
\hline
\end{tabular}

Databases: OVID MEDLINE Epub Ahead of Print, In-Process \& Other Non-Indexed Citations, Ovid MEDLINE(R) Daily and Ovid MEDLINE(R) 1946 to 7 March 2021.

\section{Literature search}

We developed a comprehensive search strategy with input from a research librarian at McMaster University (tables 1-3). The systematic search for existing relevant systematic review and original studies will be conducted in the Ovid MEDLINE and Embase (Excerpta Medica dataBASE) electronic databases and the Cochrane
Library (including Cochrane Database of Systematic Reviews and Cochrane Central Register of Controlled Trials (CENTRAL)), from inception to 7 March 2020. Search terms will cover all words related to birth control, ingredients of OCs, hormonal contraceptives, menstrual cycle and sleep. We will limit the search to studies or systematic reviews published from 1970 to the present to

\section{Table 2 Search strategy for OVID Embase}

\section{Number Searches}

1 exp contraceptive agents/ or exp hormonal contraception/ or exp contraception/ or fertility control.mp. or (fertiliz* adj3 inhibit*). $\mathrm{mp}$. or exp birth control/ or exp family planning services/ or desogestrel/ or nor pregnenes/ or progesterone congeners/ or exp ethinylestradiol/ or ethinyl estradiol.mp. or ethinylestradiol.mp. or dienogest.mp. or exp progestin/ or progestin\$.mp. or progestogen.mp. or lynestrenol/ or norethindrone/ or levonorgestrel/ or etynodiol diacetate.mp. or ethynodiol diacetate. $\mathrm{mp}$. or norgestrel or mestranol.mp. or exp mestranol/ or exp estradiol/ or estradiol.mp. or oetradiol.mp. or desogestrel.mp. or norpregnene\$ or progestins).mp. or drospirenone.mp. or lynestrenol.mp. or norethindrone.mp. or norgestrel.mp. or Etonogestrel. $\mathrm{mp}$. or gestodene.mp. or levonorgestrel.mp. or d-norgestrel.mp. or norgestimate.mp. or dienogest.mp. or contracept ${ }^{\star}$.mp. or birth control.mp. or family planning.mp. or intrauterine device.mp. or "intra uterine device".mp. exp contraceptive devices, Female/ intrauterine system.mp. or intrauterine contraceptive device.mp. or iud ${ }^{\star} . \mathrm{mp}_{\text {. or iucd }}$.mp. or exp Medroxyprogesterone/ or Depotmedroxyprogesterone.mp. or depo-medroxyprogesterone.mp. or medroxyprogesterone.mp. or Depo-provera.mp. or depo provera.mp. or (vaginal or hormon* or contraceptive) adj2 (ring or patch or injection).mp. or DPMA.mp.

2 Exp sleep/ or exp sleep-wake transition disorders/ or exp sleep deprivation/ or exp sleep wake disorders/ or exp dyssomnias/ or fragmented sleep.mp. or insufficient sleep syndrome\$.mp. or exp sleep latency/ or exp sleep hygiene/ or exp sleep arousal disorder/ or exp sleep disorder/ or sleep*.af. or insomnia\$.mp. or hypersomnia\$.mp. or "disorders of excessive somnolence" or exp restless legs syndrome/ or restless legs syndrome.mp

$\begin{array}{ll}3 & \text { (editorial or note or letter erratum or short survey or abstract).pt. or abstract report/ or letter/ or case study/ } \\ 4 & \text { animal/ not human/ } \\ 5 & \text { or/3-4 } \\ 6 & \text { exp female/ or female\$.mp. or women.mp. or woman.mp. } \\ 7 & \text { (1 and } 2 \text { and } 6 \text { ) not } 5 \\ 8 & \text { limit } 7 \text { to } y r=" 1970-c u r r e n t "\end{array}$

Database: Embase 1974-7 March 2021. 


\begin{tabular}{|c|c|}
\hline Number & Searches \\
\hline & $\begin{array}{l}\text { MeSH descriptor: [Contraceptive Agents] explode all trees or MeSH descriptor: [Hormonal Contraception] explode all trees or } \\
\text { MeSH descriptor: [Contraception] explode all trees or fertility control or fertilization inhibition or inhibition of fertilization or MeSH } \\
\text { descriptor: [Family Planning Services] explode all trees or MeSH descriptor: [Desogestrel] this term only or MeSH descriptor: } \\
\text { [Norpregnenes] this term only or MeSH descriptor: [Progesterone Congeners] this term only or MeSH descriptor: [Ethinyl } \\
\text { Estradiol] explode all trees or ethinyl estradiol or ethinylestradiol or dienogest or MeSH descriptor: [Progestins] explode all trees } \\
\text { or progestin\$ or progestogen or MeSH descriptor: [Lynestrenol] this term only or MeSH descriptor: [Norethindrone] this term } \\
\text { only or MeSH descriptor: [Norgestrel] this term only or MeSH descriptor: [Levonorgestrel] this term only or Etynodiol diacetate } \\
\text { or mestranol or MeSH descriptor: [Mestranol] explode all trees or MeSH descriptor: [Estradiol] explode all trees or estradiol or } \\
\text { oetradiol or desogestrel or norpregnenes\$ or progestins or progesterone congeners adj2 synthetic or drospirenone or lynestrenol } \\
\text { or norethindrone or norgestrel or Etonogestrel or gestogene or levonorgestrel or norgestimate or dienogest or contracept or birth } \\
\text { control or family planning or MeSH descriptor: (Intrauterine device) this term only or intrauterine device or intrauterine system or } \\
\text { intrauterine contraceptive device or iud or d-norgestrel or iucd or depotmedroxyprogesterone or depo-medroxyprogesterone or } \\
\text { medroxyprogesterone or depo-provera or depo provera or DPMA or Vaginal ring/ or ethyndiol diacetate }\end{array}$ \\
\hline 2 & $\begin{array}{l}\text { MeSH descriptor: [Sleep] explode all trees or MeSH descriptor:(Sleep-Wake Transition Disorders)explode all trees or MeSH } \\
\text { descriptor: [Sleep Deprivation] explode all trees or MeSH descriptor: [Sleep Wake Disorders] explode all trees or MeSH descriptor: } \\
\text { [Dyssomnias] explode all trees or MeSH descriptor: [Dyssomnias] explode all trees or ragmented sleep or insufficient sleep } \\
\text { syndrome\$ or MeSH descriptor: [Sleep Hygiene] explode all trees or MeSH descriptor: [Sleep Latency] explode all trees or } \\
\text { MeSH descriptor: [Sleep Arousal Disorders] explode all trees or sleep* or insomnia\$ or hypersomnia } \$ \text { or disorders of excessive } \\
\text { somnolence or MeSH descriptor: [Restless Legs Syndrome] explode all trees or restless legs syndrome }\end{array}$ \\
\hline 3 & $\begin{array}{l}\text { (comment or letter or editorial or note or erratum or short survey or news or newspaper article or patient education handout or case } \\
\text { reports or historical article).pt. }\end{array}$ \\
\hline 4 & MeSH descriptor: [Animals] explode all trees \\
\hline 5 & MeSH descriptor: [Humans] explode all trees \\
\hline 6 & 3 and 4 not 5 \\
\hline 7 & (1 and 2) not 6 \\
\hline 8 & limit 7 to $y r=" 1970$-current" \\
\hline
\end{tabular}

Database: Cochrane CENTRAL from inception to 7 March 2021.

CENTRAL, Central Register of Controlled Trials.

ensure that we include all published studies or reviews on hormonal contraceptives, excluding first-generation OCs, which had been discontinued after 1970s. For sleep outcome measures, we will search sleep terms in multipurpose fields besides the title and abstract in case sleep is investigated as a secondary outcome and therefore not reported in the abstract. The comprehensive search strategies for each database are presented in tables 1-3. The reference lists of all included studies will be checked to identify additional potentially eligible studies.

\section{Study selection and screening}

Systematic screening of the studies will be first conducted at the title and abstract levels and then at the full-text level to determine if a study meets eligibility criteria by two independent reviewers. Any potential conflicts between the reviewers will be resolved through discussion. If discrepancies in judgement remain after discussion, a third-party reviewer will be consulted to resolve the conflict and to provide a final decision.

\section{Eligibility criteria}

Study characteristics

Existing systematic reviews will be included if they (1) address the same research question with similar inclusion and exclusion criteria as ours and (2) have a low risk of bias in study eligibility criteria, identification and selection of studies, data collection and study appraisal, and synthesis and findings assessed using the Risk of Bias in Systematic Reviews (ROBIS) tool. ${ }^{27}$

For original studies, we will include (1) randomised controlled trials and non-randomised comparative studies that take into account the effect of potential confounders (eg, age, race and socioeconomic status) ${ }^{28-30}$ using methods such as multivariable analysis, propensity-score matching or showing no statistically significant differences in baseline characteristics between participants in comparison groups; (2) studies on human participants; and (3) studies which investigate the association between hormonal contraceptive use and sleep-related outcomes, either as the primary or the secondary objective.

\section{Participants}

Participants or subgroups of participants are female, between 15 and 49 years old.

\section{Exposure}

Participants who take second-generation or more recent OC or other hormonal contraceptives containing both progesterone and oestrogen or progesterone only, regardless of its brand, dose and frequency, to avoid pregnancy, as long as their hormonal contraceptive use pattern is sustained for at least 3 months by the time sleep outcomes are assessed. The first 3 months of hormonal contraception use or discontinuation of hormonal contraception are excluded to account for altered menstruation that 
occurs following discontinuation of hormonal contraception and significant mood changes seen with initiation of hormonal contraception. ${ }^{31} 32$

\section{Comparator}

Included studies must have comparison groups of women who are using (1) non-hormonal contraceptives; (2) naturally cycling (ie, not use any contraceptive methods) or placebo; (3) same agent as the exposure but in different dose, frequency and duration; and (4) second-generation or more recent OC or other hormonal contraceptives different from the exposure. Since recent usage or cessation of hormonal contraceptive use may cause hormonal oscillations, we will only include studies with participants keeping their contraceptive use pattern (either using different hormonal contraceptives from the exposure or placebo, or not using any hormonal contraceptive) for at least 3 months by the time sleep outcomes are assessed.

\section{Outcomes}

We are interested in any sleep-related outcomes assessed at any time points after keeping their contraceptive use pattern for at least 3 months. A variety of sleep measurements for sleep quality, sleep duration and sleep disorder will be included in this systematic review. The sleep measurements can be questionnaires based on Likert Scale items listed as follows but not limited to these tools: 1. The Pittsburgh Sleep Quality Index (PSQI) ${ }^{33}$ It measures seven domains: subjective sleep quality, sleep latency, sleep duration, habitual sleep efficiency, sleep disturbances, use of sleep medications and daytime dysfunction over the last 4 weeks. The PSQI is a subjective measure of sleep. Subjects self-rate each of the seven domains of sleep from 0 to 3, where three represents the negative extreme on the Likert Scale. A subject with a global sum of 5 or greater is considered as a poor sleeper. Studies reported sleep quality in any of the aforementioned seven domains during and after taking hormonal contraceptives will be included in the review.

2. The Epworth Sleepiness Scale is a self-administered test used to assess daytime sleepiness. ${ }^{34}$ It consists of eight questions with different scenarios and a scale from 0 to 3 where subjects would indicate how likely they are to fall asleep in that situation. The scores are summed for a total score between 0 and $24 .{ }^{34}$ Studies reporting sleepiness with any of the eight questions during and after taking hormonal contraceptives will be included in the review.

3. The Athens Insomnia Scale (AIS), which is also a selfadministered psychometric instrument designed for quantifying sleep difficulty. ${ }^{35}$ The AIS consists of eight items: sleep induction (ie, time to fall asleep after turning off the lights), night awakenings, final awakening earlier than desired, total sleep duration, overall quality of sleep, sense of well-being during the day, functioning (physical and mental) during the day and sleepiness during the day. Each item is rated from 0 to
3 with higher scores indicating more impaired sleep. The total score ranges from 0 to 24 , and a total score of 6 or more is considered as insomnia. Studies which report any or all of the aforementioned eight insomniarelated items during and after taking hormonal contraceptives will be included in the review.

4. The Insomnia Severity Index (ISI), a self-administered questionnaire used to assess insomnia. ${ }^{36}$ The ISI consists of a 1-month recall period and assesses seven domains: severity of sleep onset, sleep maintenance, difficultly waking up in the morning, sleep dissatisfaction, interference of sleep with daytime functioning, noticeability of sleep problems by others and distress caused by sleep difficulties. Each domain is rated on a scale from 0 to 4 with higher scores indicating more problematic sleep. The score is summed forming a total score between 0 and 28, with a score of 8 and above indicative of insomnia. ${ }^{36}$ Studies reporting any domains from the ISI during and after taking hormonal contraceptives will be included in the review.

We will also include continuous measures of sleep quality and disorder. These measures include (1) total amount of sleep obtained, either during nocturnal sleep episode or across the 24-hour period; (2) sleep latency, that is, how long it takes the participant to fall asleep; (3) how many times the participant wakes up; (4) how many minutes the participant is awake during the night; and (5) sleep efficiency, that is, what percentage of time spent in bed the participant is actually asleep. The total sleep time is equal to the total amount of time spent in bed (in minutes) minus the sum of time it takes to fall asleep, and the time spent awake throughout the night. The percentage is calculated as the total sleep time divided by total time in bed. Given we will only include studies with participants keeping their contraceptive use pattern for at least 3 months, the sleep outcomes from the eligible studies will be summarised after participants keeping their contraceptive use pattern for at least 3 months as well.

\section{Exclusion criteria}

The following studies will be excluded from this systematic review: (1) studies only recruited patients with specific comorbidities or conditions, such as hormonal disorder, acne, perimenopause, postmenopause, dysmenorrhea, depression, etc, since these conditions may be associated with sleep problems and will not allow us to elucidate the association between hormonal contraceptives and sleep; (2) studies investigating the first-generation OC since it is not available in the market anymore due to severe side effects; (3) studies investigating emergency contraception; and (4) studies in languages other than English. We will exclude emergency contraception from this review as the hormonal effect of the emergency contraception is significantly different from long-acting contraception. The half-life of oral leovnoregestrel is around 24-32 hours, which is not significant when considering long-term effect on sleep. ${ }^{37}$ 


\section{Data abstraction}

A standard form will be developed to extract data from the included studies. From each article, the author, study design, study population, publication year, journal, participant demographics, hormonal contraceptive used, intervention details, comparison groups, sleep outcomes, and the association between hormonal contraceptive use and sleep will be extracted. Additionally, any mean values, SD and CIs, and all information needed for appraisal of internal validity will be extracted. This will be done in a standardised data extraction form by two reviewers independently. When consensus is not reached, a third researcher will be consulted to reach the final decision. Data will be presented in narrative form and summary tables.

\section{Risk of bias assessment}

For randomised controlled trials, the risk of bias in each included study for each outcome will be evaluated independently by two reviewers using the Cochrane Collaboration's Risk of Bias for Randomised Trials V.2.0 tool. ${ }^{38}$ Studies will be assessed from five domains: bias from randomisation, bias from deviates from intended interventions, bias due to missing data, bias in outcome measurement and bias in the selection of the reported result. ${ }^{38}$ An overall rating of low bias, some concerns about bias or high bias will be given, depending on the result of assessment. The ROBINS-I tool will be used to assess the risk of bias for each outcome in non-randomised comparative studies. ${ }^{38}$ Studies will be assessed from seven domains: bias due to confounding, bias in selection of participants into the study, bias in classification of interventions, bias due to deviations from intended interventions, bias due to missing data, bias in measurement outcomes and bias in selection of the reported result. ROBIS tool will be used to assess the risk of bias for the eligible systematic reviews. ${ }^{39}$ Reviews will be assessed from four domains: study eligibility criteria, identification and selection of studies, data collection and study appraisal, and synthesis and findings. We will also assess the overall risk of bias in the interpretation of review findings and whether this considered limitations identified in any of the aforementioned four domains.

\section{Grading the strength of evidence}

The certainty of the evidence per outcome for each comparison will be assessed from five domains: risk of bias, inconsistency, indirectness, imprecision and publication bias using the Grading of Recommendations, Assessment, Development, and Evaluation (GRADE) approach and be classified into four levels of evidence (high, moderate, low or very low). ${ }^{40}$

\section{Data synthesis}

All data extracted will be presented in both narrative form and in summary tables. If data are missing, the study authors will be contacted to attempt to obtain the data. For sleep measures investigated by two or more studies, if there is no clinical heterogeneity (eg, different age or intervention) and methodological heterogeneity (eg, different measurement tools), statistical heterogeneity will be assessed using forest plot visually, the $\chi^{2}$ test of homogeneity ( $p$ value $<0.05$ ), and quantified using the Higgins' $\mathrm{I}^{2}$ statistic with $25 \%, 50 \%$ and $75 \%$ representing low, medium and high heterogeneities, respectively. ${ }^{41}$ The presence of publication bias will be evaluated using a funnel plot and the Duval and Tweedie's trim and fill method. ${ }^{42}$

We will use frequentist approach to estimate the overall effect of hormonal contraceptives on sleep. For binary outcome measures, we will express the results of each study as a risk ratio (RR) and its $95 \%$ CI. We will perform meta-analyses of pooling the RRs with $95 \%$ CIs of studies using a random-effects model, because of anticipated heterogeneity of hormonal contraceptives, study designs and participants. For continuous outcome measures, standardised mean differences (SMDs) will be used for the final assessment from individual studies due to the likely variability in the measuring scales. The SMD will be categorised as small, medium and large based on the thresholds $0.2,0.5$ and 0.8 , respectively, as suggested by Cohen. ${ }^{43}$ The $95 \%$ CI will be used to represent the deviation from the point estimate for both the individual studies and the pooled estimate. Random-effects metaanalysis will be used to obtain the pooled estimates. If sleep outcomes are measured at multiple time points in a study, the measure done at the similar time point as the other studies will be used in the primary metaanalysis. The outcome measured at other time points will be considered in the subgroup analysis by duration of hormonal contraceptives.

We will run subgroup analyses by hormonal method of contraception (implant, IUD, injections, pills, vaginal rings or skin patches), agent (progesterone and oestrogen combined or progesterone only), dose and duration of contraceptives. We will run sensitivity analyses to assess whether our conclusions will be robust if excluding studies with high risk of bias and non-randomised comparative studies. As long as studies included in the systematic review form a connected network, a network meta-analysis using both direct and indirect evidence will be conducted to estimate the comparative harm of different contraceptives. ${ }^{45}$ Results from the network meta-analysis will allow us to summarise and interpret a wider picture of the evidence for the putative effect of hormonal contraceptives on sleep. We will conduct data analysis using the Cochrane Collaboration Review Manager statistical software (V.5.4.1) (http://ims.cochrane.org/RevMan).

\section{Patient and public involvement}

There is no patient or public involvement in this study.

\section{Ethics and dissemination}

Ethical approval is not required for this protocol nor the subsequent review, as this review consists only of an analysis of previously published works. The findings of 
this review will be published in a peer-reviewed journal and disseminated in conferences related to this topic. All amendments to this protocol will be documented in the publication of the review.

\section{DISCUSSION}

\section{Strengths and limitations}

This systematic review will be the first to provide quantitative estimates of the association between hormonal contraceptive use and sleep outcomes in healthy women. Our systematic review methodology includes explicit eligibility criteria created in consultation with research librarians, and the usage of tested, standardised abstraction forms. We will calibrate our review methods throughout the review process for optimal consistency among reviewers. The GRADE approach will be applied to aggregate data; the Preferred Reporting Items for Systematic Reviews and Meta-Analyses for Protocols reporting checklist is followed to draft this systematic review protocol; and the Preferred Reporting Items for Systematic Reviews and Meta-Analyses reporting checklist will be followed to draft this systematic review later. The $\kappa$ statistic will be reported to identify issues with reproducibility. Our review will encompass a wide range of hormonal contraceptive formulations and sleep outcome measurements. We anticipate limitations with regard to publication bias and recall bias as the majority of studies employ selfadministered tools to assess sleep quality and duration.

\section{Study implications}

Sleep quality is a crucial outcome that affect overall life quality in women of reproductive age and may indicate risk of developing comorbid conditions. The relationship between hormonal contraceptive use and sleep quality, duration and disorder have been reported inconsistently in the current literature. Hormonal contraceptives have been more and more widely accepted by women. However, no study has attempted to provide systematic evidence on the association between hormonal contraceptive use and sleep. Our systematic review will elucidate the association between hormonal contraceptive use and sleep quality. Moreover, it will contribute evidence that will support the improvement of guidelines for taking hormonal contraceptive in healthy women and promote awareness of safety for taking hormonal contraceptive.

It is challenging to design, conduct and analyse original studies investigating the association between hormonal contraceptive use and sleep quality. For instance, a wide variety of hormonal contraceptives are used by women who may change to other brands of contraceptive or birth control methods. In addition, most studies use subjective sleep measures, which may involve recalling the information in the past. Through appraisal of published studies, our systematic review will inform the improvement in the design and implementation of future research in this field.
Author affiliations

${ }^{1}$ Department of Health Research Methods, Evidence, and Impact, McMaster University, Hamilton, Ontario, Canada

${ }^{2}$ Faculty of Health Sciences, McMaster University, Hamilton, Ontario, Canada ${ }^{3}$ Department of Obstetrics and Gynecology, Peking University Third Hospital, Beijing, China

${ }^{4}$ Department of Neuropsychiatry and Behavioral Neurology, Beijing Tiantan Hospital, Capital Medical University, Beijing, China

${ }^{5}$ Institute of Sleep and Consciousness Disorders,Beijing Institute of Brain Disorders, Collaborative Innovation Center for Brain Disorders, Capital Medical University, Beijing, China

${ }^{6}$ Faculty of Science, McMaster University, Hamilton, Ontario, Canada

${ }^{7}$ Center for Clinical Practice Guideline Conduction and Evaluation, Children's Hospital of Fudan University, Shanghai, China

Contributors JM, MC, XY and NZ conceptualised and designed the protocol, drafted the initial manuscript and reviewed the manuscript. MC, XY, JM, QW, HK and HR defined the concepts and search items, data extraction process as well as methodological appraisal of the study. MC, JM, QW and XY planned the data extraction and statistical analysis. LT, CM and CW provided critical insights. All authors approved the final manuscript.

Funding This research is supported by the National Key Research and Development Program of China (number 2020YFC2005304) and Beijing Key Clinical Specialty Construction Project.

Competing interests None declared.

Patient and public involvement Patients and/or the public were not involved in the design, conduct, reporting or dissemination plans of this research.

Patient consent for publication Not applicable.

Provenance and peer review Not commissioned; externally peer reviewed.

Open access This is an open access article distributed in accordance with the Creative Commons Attribution Non Commercial (CC BY-NC 4.0) license, which permits others to distribute, remix, adapt, build upon this work non-commercially, and license their derivative works on different terms, provided the original work is properly cited, appropriate credit is given, any changes made indicated, and the use is non-commercial. See: http://creativecommons.org/licenses/by-nc/4.0/.

\section{ORCID iDs}

Jinhui Ma http://orcid.org/0000-0002-8033-1699

Megan Cheng http://orcid.org/0000-0003-1248-8034

Lehana Thabane http://orcid.org/0000-0003-0355-9734

Caihong Ma http://orcid.org/0000-0003-0439-9621

Xiaomei Yao http://orcid.org/0000-0002-1210-3758

\section{REFERENCES}

1 United Nations. Contraceptive use by method 2019: data booklet, 2019. Available: https://www.un-ilibrary.org/population-anddemography/contraceptive-use-by-method-2019_1bd58a10-en [Accessed 20 Jul 2020].

2 van den Heuvel MW, van Bragt AJM, Alnabawy AKM, et al. Comparison of ethinylestradiol pharmacokinetics in three hormonal contraceptive formulations: the vaginal ring, the transdermal patch and an oral contraceptive. Contraception 2005;72:168-74.

3 Dean G, Schwarz EB. "Intrauterine contraceptives (IUCs)". In: Hatcher RA, Trussell J, Nelson AL, et al, eds. Contraceptive technology. 20 edn. New York: Ardent Media, 2011: 147-91.

4 Lancel M, Faulhaber J, Schiffelholz T, et al. Allopregnanolone affects sleep in a benzodiazepine-like fashion. $J$ Pharmacol Exp Ther 1997;282:1213-8.

5 Allada R, Cirelli C, Sehgal A. Molecular mechanisms of sleep homeostasis in flies and mammals. Cold Spring Harb Perspect Biol 2017;9:a027730.

6 Schwartz MD, Mong JA. Estradiol modulates recovery of REM sleep in a time-of-day-dependent manner. Am J Physiol Regul Integr Comp Physiol 2013;305:R271-80.

7 Bezerra AG, Andersen ML, Pires GN, et al. Effects of hormonal contraceptives on sleep - possible treatment for insomnia in premenopausal women. Sleep Sci 2018;11:129-36.

8 Léger D, Bayon V. Societal costs of insomnia. Sleep Med Rev 2010;14:379-89.

9 Stoller MK. Economic effects of insomnia. Clin Ther 1994;16:873-97. 
10 Gourineni R. Prognosis and complications. In: Attarian HP, ed. Clinical handbook of insomnia. 3rd edn. New York: Springer, 2017: 59-73.

11 Kessler RC, Berglund PA, Coulouvrat C, et al. Insomnia and the performance of US workers: results from the America insomnia survey. Sleep 2011;34:1161-71.

12 Ohayon MM, O'Hara R, Vitiello MV. Epidemiology of restless legs syndrome: a synthesis of the literature. Sleep Med Rev 2012;16:283-95.

13 Wimms A, Woehrle H, Ketheeswaran S, et al. Obstructive sleep apnea in women: specific issues and interventions. Biomed Res Int 2016;2016:1-9.

14 Reed BG, Carr BR. The normal menstrual cycle and the control of ovulation. In: Endotext. South Dartmouth (MA): MDText.com, Inc, 2000. http://www.ncbi.nlm.nih.gov/books/NBK279054/

15 Baker FC, Lee KA. Menstrual cycle effects on sleep. Sleep Med Clin 2018;13:283-94.

16 Rivera R, Yacobson I, Grimes D. The mechanism of action of hormonal contraceptives and intrauterine contraceptive devices. Am J Obstet Gynecol 1999;181:1263-9.

17 Gierisch JM, Coeytaux RR, Urrutia RP, et al. Oral contraceptive use and risk of breast, cervical, colorectal, and endometrial cancers: a systematic review. Cancer Epidemiol Biomarkers Prev 2013;22:1931-43.

18 Helmerhorst FM, Bloemenkamp KW, Rosendaal FR, et al. Oral contraceptives and thrombotic disease: risk of venous thromboembolism. Thromb Haemost 1997;78:327-33.

19 van Hylckama Vlieg A, Helmerhorst FM, Rosendaal FR. The risk of deep venous thrombosis associated with injectable depotmedroxyprogesterone acetate contraceptives or a levonorgestrel intrauterine device. Arterioscler Thromb Vasc Biol 2010;30:2297-300.

20 Leblanc ES, Laws A. Benefits and risks of third-generation oral contraceptives. J Gen Intern Med 1999;14:625-32.

21 Iversen L, Sivasubramaniam S, Lee AJ. Lifetime cancer risk and combined oral contraceptives: the royal college of general practitioners' oral contraception. Am J Obstet Gynecol 2017;216:580.e1-580.e9.

22 Guida M, Rega A, Vivone I, et al. Variations in sleep associated with different types of hormonal contraceptives. Gynecol Endocrinol 2020;36:166-70.

23 Hachul H, Andersen ML, Bittencourt L, et al. A population-based survey on the influence of the menstrual cycle and the use of hormonal contraceptives on sleep patterns in São Paulo, Brazil. Int J Gynaecol Obstet 2013;120:137-40.

24 Lundin C, Danielsson KG, Bixo M, et al. Combined oral contraceptive use is associated with both improvement and worsening of mood in the different phases of the treatment cycle double-blind, placebo-controlled randomized trial. Psychoneuroendocrinology 2017;76:135-43.

25 Bezerra AG, Andersen ML, Pires GN, et al. Hormonal contraceptive use and subjective sleep reports in women: an online survey. $J$ Sleep Res 2020;29:e12983.

26 Moher D, Shamseer L, Clarke M, et al. Preferred reporting items for systematic review and meta-analysis protocols (PRISMA-P) 2015 statement. Syst Rev 2015;4:1.
27 Whiting P, Savović J, Higgins JPT, et al. ROBIS: a new tool to assess risk of bias in systematic reviews was developed. $J$ Clin Epidemiol 2016;69:225-34.

28 Jones J, Mosher W, Daniels K. Current contraceptive use in the United States, 2006-2010, and changes in patterns of use since 1995. Natl Health Stat Report 2012;60:1-25.

29 Rao U, Hammen CL, Poland RE. Ethnic differences in electroencephalographic sleep patterns in adolescents. Asian $\mathrm{J}$ Psychiatr 2009;2:17-24.

30 Grandner MA, Patel NP, Gehrman PR, et al. Who gets the best sleep? ethnic and socioeconomic factors related to sleep complaints. Sleep Med 2010;11:470-8.

31 Nassaralla CL, Stanford JB, Daly KD, et al. Characteristics of the menstrual cycle after discontinuation of oral contraceptives. J Womens Health 2011;20:169-77.

32 Schaffir J, Worly BL, Gur TL. Combined hormonal contraception and its effects on mood: a critical review. Eur J Contracept Reprod Health Care 2016;21:347-55.

33 Buysse DJ, Reynolds CF, Monk TH, et al. The Pittsburgh sleep quality index: a new instrument for psychiatric practice and research. Psychiatry Res 1989;28:193-213.

34 Johns MW. A new method for measuring daytime sleepiness: the epworth sleepiness scale. Sleep 1991;14:540-5.

35 Soldatos CR, Dikeos DG, Paparrigopoulos TJ. Athens insomnia scale: validation of an instrument based on ICD-10 criteria. $J$ Psychosom Res 2000;48:555-60.

36 The Insomnia Severity Index. Psychometric indicators to detect insomnia cases and evaluate treatment response. Available: https:// www.ncbi.nlm.nih.gov/pmc/articles/PMC3079939/ [Accessed 20 Jul 2020].

37 Kuhl H. Pharmacology of estrogens and progestogens: influence of different routes of administration. Climacteric 2005;8 Suppl 1:3-63.

38 Sterne JAC, Savović J, Page MJ, et al. Rob 2: a revised tool for assessing risk of bias in randomised trials. BMJ 2019;366:14898.

39 Whiting P, Savović J, Higgins JPT, et al. Robis: a new tool to assess risk of bias in systematic reviews was developed. $J$ Clin Epidemiol 2016;69:225-34.

40 Schünemann HJ, Brożek J, Guyatt G. Grade handbook, 2013. Available: https://gdt.gradepro.org/app/handbook/handbook.html [Accessed 28 Sep 2020].

41 Higgins JPT, Thompson SG, Deeks JJ, et al. Measuring inconsistency in meta-analyses. BMJ 2003;327:557-60.

42 Duval S, Tweedie R. Trim and fill: a simple funnel-plot-based method of testing and adjusting for publication bias in meta-analysis. Biometrics 2000;56:455-63.

43 Cohen J. Statistical power analysis for the behavioral sciences. 2nd edn. Hillsdale, NJ: Lawrence Erlbaum Associates, 1998.

44 Chaimani A, Caldwell DM, Li T. Chapter 11: Undertaking network meta-analyses. In: Cochrane handbook for systematic reviews of interventions, 2019.

45 Caldwell DM, Welton NJ. Approaches for synthesising complex mental health interventions in meta-analysis. Evid Based Ment Health 2016;19:16-21. 